\title{
On Scheduling Problems with Applications to Packet-Switched Optical WDM Networks *
}

\author{
Evripidis Bampis George N. Rouskas \\ TR-2000-07
}

July 18, 2000

\begin{abstract}
We consider a scheduling problem, which we call the Scheduling and Wavelength Assignment (SWA) problem, arising in optical networks that are based on the Wavelength Division Multiplexing (WDM) technology. We prove that the SWA problem is $\mathcal{N} \mathcal{P}$-hard for both the preemptive and the non-preemptive cases. Furthermore, we propose two efficient approximation algorithms. The first is for the preemptive case and it is based on a natural decomposition of the problem to the classical multiprocessor scheduling and open-shop problems. For the non-preemptive case, we prove that a naive implementation of list scheduling produces a schedule that can be $m$ times far from the optimum, where $m$ is the number of processors (equivalently, WDM channels). Finally, we give a more refined version of list scheduling and we prove it to be a 2-approximation algorithm for both the off-line and the on-line contexts.
\end{abstract}

Department of Computer Science

North Carolina State University

Raleigh, NC 27695-7534

*This work was performed while G. N. Rouskas was visiting the Université d'Evry Val d'Essone. The work of G. N. Rouskas was supported by the Université d'Evry Val d'Essonne, by the National Science Foundation under grant NCR-9701113 (CAREER), and by the Defense Advanced Research Projects Agency under grant F-30602-00-C-0034. 


\section{Introduction}

The spectacular growth in data traffic and the surging demand for diverse services has led to a dramatic increase in demand for data transmission capacity. Recent advances in wavelength division multiplexing (WDM) technology [5, 7] are expected to provide solutions to this challenge. WDM supports multiple simultaneous channels, each on a different wavelength, on a single fiber. WDM systems operating at aggregate rates exceeding one Terabit per second have been demonstrated, while systems supporting rates of tens of Gigabits per second are becoming commercially available.

As future networks based on WDM technology are developed to support data traffic and the Internet, they must be designed and optimized for that purpose. In particular, a number of new and challenging problems arise in the area of scheduling data packets over multiple wavelengths, both in a local area environment [17] and in a backbone network consisting of IP routers [15]. In this paper we consider a scheduling problem with applications to packet-switched optical WDM networks, and we prove that it is $\mathcal{N} \mathcal{P}$-hard for both the preemptive and the non-preemptive cases. We then present two efficient approximation algorithms for this problem. For the preemptive case, the approximation algorithm is based on a natural decomposition of the problem into the classical multiprocessor scheduling and open-shop problems. For the non-preemptive case, we develop two list scheduling algorithms, the second of which is a 2-approximation algorithm for both the on-line and off-line contexts.

The paper is organized as follows. In the next section we define the scheduling problem under study, and we motivate it by describing its relationship to packet scheduling in WDM optical networks. In Section 3 we present an off-line approximation algorithm for preemptive scheduling, and in Section 4 we present both off-line and on-line algorithms for the non-preemptive case. We conclude the paper in Section 5.

\section{Problem Definition and Applications}

\subsection{The Scheduling and Wavelength Assignment (SWA) Problem}

We consider a set $\mathcal{M}$ of $m$ processors and a set $\mathcal{J}$ of $n, n>m$ jobs. Each job $J_{d} \in \mathcal{J}$ is defined as a set of $n$ operations, $J_{d}=\left\{O_{1 d}, O_{2 d}, \cdots, O_{n d}\right\}$, and $p_{s d}$ denotes the processing time of operation $O_{s d}$. The objective is to schedule the $n$ jobs on the $m$ processors so as to minimize the makespan, or maximum finish time of the schedule, subject to the following constraints:

C1. All operations of job $J_{d}$ are executed on the same processor.

C2. The operations $O_{s d}$ and $O_{s d^{\prime}}$ cannot be simultaneously executed, for all $s, d \neq d^{\prime}$.

C3. A processor may execute at most one operation at any time instant.

C1 and C2 define a set of compatibility constraints among the different operations. Specifically, two operations $O_{s d}$ and $O_{s^{\prime} d^{\prime}}$ are said to be incompatible if either $s=s^{\prime}$ or $d=d^{\prime}$; otherwise the operations 
are compatible. Incompatible operations cannot be executed simultaneously. Constraint $\mathbf{C 1}$ also implies that once an operation of job $J_{d}$ has been executed on a processor $x$, then all operations $O_{s d}, s=1, \cdots, n$, must be executed on the same processor $x$. However, the processor on which the operations of a job $J_{d}$ are executed is not known in advance, rather, it is determined as part of the solution to the scheduling problem. Furthermore, the operations of a job $J_{d}$ can be executed on processor $x$ in any order.

The scheduling problem defined above can be logically decomposed into two sub-problems. Because of constraint $\mathbf{C 1}$, the first sub-problem is to assign each job $J_{d}$ to a processor $x$, meaning that all operations of $J_{d}$ will be executed on $x$. Given this assignment of jobs to processors, the second sub-problem is to schedule the operations on their assigned processors so as to minimize the makespan, while also satisfying the compatibility constraints $\mathbf{C 2}$ and the processor constraint C3. This decomposition leads to a natural way of solving the scheduling problem by applying existing algorithms to each sub-problem in isolation, as discussed later. However, we will also show that it is possible to design algorithms to simultaneously solve both sub-problems, and these algorithms are more efficient than the two-step approach described above.

We will refer to this type of scheduling problem as the Scheduling and Wavelength Assignment (SWA) problem, since it arises naturally in packet-switched optical WDM networks, as we explain in Section 2.3. We have also chosen to use $s$ and $d$ as subscripts for the operations to reflect the fact that, in the network settings described in Section 2.3, operation $O_{s d}$ corresponds to the amount of traffic to be transmitted from a source $s$ to a destination $d$ in the network.

We now introduce notation that will be useful in later sections. Let $T_{s e q}$ denote the sum of the computation times of all operations, let $D_{\max }$ be the maximum amount of computation time required by any job, and let $S_{\max }$ denote the maximum amount of computation time associated with any source $s$ :

$$
\begin{gathered}
T_{\text {seq }}=\sum_{s=1}^{n} \sum_{d=1}^{n} p_{s d} \\
D_{\text {max }}=\max _{d=1, \cdots, n}\left\{\sum_{s=1}^{n} p_{s d}\right\} \\
S_{\text {max }}=\max _{s=1, \cdots, n}\left\{\sum_{d=1}^{n} p_{s d}\right\}
\end{gathered}
$$

Let $C_{m a x}^{o p t}$ denote the optimal makespan. We obviously have that:

$$
\begin{aligned}
& C_{\max }^{\text {opt }} \geq \frac{T_{\text {seq }}}{m} \\
& C_{\max }^{\text {opt }} \geq S_{\max } \\
& C_{\max }^{\text {opt }} \geq D_{\max }
\end{aligned}
$$

\subsection{Related Classical Scheduling Problems}

There are two classical scheduling problems that are closely related to the $S W A$ problem we consider in this work. The first one is the multiprocessor scheduling problem $[6,11]$, in which we have a set of $n$ tasks 
that must be scheduled on $m$ machines in such a way that the makespan is minimized. As in the $S W A$ problem, the tasks can be executed on any machine and their processing times are not machine-dependent. It is well-known that the multiprocessor scheduling problem is $\mathcal{N} \mathcal{P}$-hard [6], and that any list-scheduling algorithm is a 2-approximation algorithm [10]. Note that this result is very important, since it remains valid in an on-line context. Of course, for the off-line case, there exist algorithms which provide significantly better performance guarantees. These include the LPT (Largest Processing Time) algorithm, which is a 4/3approximation algorithm, and the MULTI-FIT algorithm, which guarantees a relative performance of 1.2 [4]. In addition, there exists a polynomial time approximation scheme (PTAS) for the multiprocessor scheduling problem that is due to Hochbaum and Schmoys [12]. The main difference between the multiprocessor scheduling problem and the $S W A$ problem is that in the former, the $n$ tasks are assumed to be pair-wise independent, and thus, any two tasks may be scheduled simultaneously on different processors. In the $S W A$ problem, on the other hand, there is a set of compatibility constraints among the operations, as defined by constraints $\mathbf{C 1}$ and $\mathbf{C 2}$, which prevent the simultaneous execution of certain operations.

The second related problem is the open-shop problem where we have a set of $n$ jobs with $m$ operations each $[11,9,14,8]$, such that the $l$-th operation, $l=1, \cdots, m$, of a job must be processed on machine $l$. Similar to the $S W A$ problem, under open-shop scheduling, the operations of any given job may be processed in any order. The preemptive version of the open-shop problem is solvable in polynomial time [9]. A polynomialtime algorithm also exists for the non-preemptive open-shop problem with $m=2$ processors [9]. However, the general non-preemptive open-shop problem is known to be $\mathcal{N} \mathcal{P}$-hard, and it has been shown that any list-scheduling algorithm is a 2-approximation algorithm [13]. Here also, it is interesting to point out that this result holds in the on-line context.

\subsection{Applications}

We now describe several network environments where the $S W A$ problem arises.

Broadcast WDM optical networks. Consider a WDM optical network with $n$ nodes interconnected via a broadcast star that supports $m<n$ distinct wavelengths [16]. Nodes communicate by exchanging fixed-length packets, and the time it takes to transmit a packet is taken as the unit of time. Since there are fewer wavelengths than nodes, packet transmissions by several nodes may share a single wavelength, and the problem of scheduling these packet transmissions arises. At the same time, an important objective in such a network is load balancing across the different wavelengths, since it has been shown that network performance deteriorates significantly if the traffic load concentrates on a few wavelengths $[18,19,1,2]$.

Let us assume that the long-term traffic requirements of the nodes are known, let $O_{s d}$ denote the operation of transmitting packets from node $s$ to node $d$, and let $p_{s d}$ be the number of packets that need to be transmitted between these two nodes in the network. Let us also assume that the nodes are equipped with fast-tunable transmitters, so that no cost is incurred when a transmitter switches from one wavelength to another, but that receivers are fixed-tuned to a certain wavelength. Let us also consider the operations of transmitting packets to a single receiver $d$ as a job $J_{d}$. Then, scheduling the packet transmissions over 
the $m$ wavelengths is equivalent to the preemptive $S W A$ problem, since the transmission of the $p_{s d}$ packets of operation $O_{s d}$ can be preempted (at the end of any packet) and continued at a later time. Note that, minimizing the makespan for this problem implies balancing the traffic across the various wavelengths by properly assigning the fixed-tuned receivers to wavelengths.

WDM IP routers employing multi-protocol label switching (MPLS). Consider a backbone network of $n$ high-speed IP routers interconnected by fiber lines each supporting $m$ distinct wavelengths. The routers employ MPLS [3], whereby each IP packet carries a special label used by the routers to forward the packet to its destination backbone router, avoiding computationally expensive IP table lookups. To avoid packet reordering at the destination router, packets for a given destination $d$ (i.e., those carrying the same label) must be sent over the same wavelength in a first-come, first-served order. Since IP packets are of variable length, a non-preemptive $S W A$ problem arises in this case.

Supporting packet traffic over WDM SONET/SDH rings. In this scenario, a node on a SONET/SDH ring must transmit ATM fixed-size cells or IP variable-size packets over the $m$ wavelengths in the ring. Assuming that there are $n$ destination nodes and that traffic to each destination must be carried on the same wavelength, the traffic scheduling problem is equivalent to the $S W A$ problem.

While all the applications described in this section are in packet scheduling in WDM networks, for the rest of the paper we will use terminology from the multiprocessor scheduling literature. The reader should keep in mind, however, that the terms "processor," "computation time," and "job" correspond to "wavelength," "transmission time," and "destination" in the network environment.

\section{$3 \quad$ Preemptive Scheduling}

Let us first assume that the operations $O_{s d}$ of any job $J_{d}$ are preemptable, i.e., there is no cost in preempting an operation and resuming it later on the same processor (refer to constraint C1). We have the following result.

Lemma 1 The preemptive $S W A$ problem is $\mathcal{N} \mathcal{P}$-complete.

Proof. Consider an instance of the $S W A$ problem with $m=2$ processors and $n$ jobs. Each job $J_{d}, d=1, \cdots, n$, of the instance consists of a single operation $O_{d d}$ with non-zero processing time $p_{d d}$ (i.e., all other operations $O_{s d}, s \neq d, s=1, \cdots, n$, of job $J_{d}$ are such that $p_{s d}=0$ ). Since an operation may not change processor after a preemption, this special case of the preemptive $S W A$ is equivalent to the PARTITION problem [6], which is $\mathcal{N} \mathcal{P}$-complete.

An approximation algorithm for the preemptive $S W A$ problem may now be obtained by considering the problem decomposition described previously. The approximation algorithm consists of two steps. The first step assigns jobs to processors in a way that attempts to balance the amount of work (i.e., the total processing time) assigned to each processor. The second step is to apply existing approximation algorithms 
to the resulting open-shop problem.

Algorithm DA (Decomposition algorithm)

S1. Let $P_{d}$ be the total processing time required by job $J_{d}, P_{d}=\sum_{s=1}^{n} p_{s d}$. Considering each job $J_{d}$ as an independent task with processing time equal to $P_{d}$, run an approximation algorithm for the resulting multiprocessor problem to assign each job $J_{d}$ to a processor.

S2. Let $\mathcal{J}_{x}$ denote the set of jobs assigned to processor $x$ at the end of Step S1. For each processor $x$, create new operations $O_{s x}^{\prime}$ with processing time $p_{s x}^{\prime}=\sum_{d \mid J_{d} \in \mathcal{J}_{x}} p_{s d}$. Now, the original $S W A$ problem has been transformed to a preemptive open-shop scheduling problem with $m$ processors and operations $O_{s x}^{\prime}$. Run the Gonzalez and Sahni algorithm [9] to obtain an optimal schedule for the new open-shop problem.

Lemma 2 Algorithm DA is an approximation algorithm for the preemptive SWA problem.

Proof. Correctness. Step $\mathbf{S} 1$ assigns each job to a certain processor, thus, all operations of a job will be executed on the same processor, satisfying constraint C1. In Step S2, a preemptive open-shop problem is constructed, such that the processing time of an operation $O_{s x}$ for a source $s$ on processor $x$ is the sum of the processing times of operations $O_{s d}$ for which job $J_{d}$ has been assigned to processor $x$. Consider two jobs $J_{d}$ and $J_{d^{\prime}}, d \neq d^{\prime}$. If the jobs have been assigned to two different processors, the Gonzalez and Sahni algorithm ensures that constraint $\mathbf{C 2}$ is satisfied. If the jobs have been assigned to the same processor, constraint C2 is also satisfied since, whenever operation $O_{s x}$ is being processed, at most one of operations $O_{s d}$ or $O_{s d^{\prime}}$ (which are part of $O_{s x}$ ) is processed, but not both. Finally, the operation of the Gonzalez and Sahni algorithm does not violate constraint C3.

Approximation claim. Consider the preemptive open-shop problem in Step S2, and let $D_{\text {max }}^{\prime}$ (respectively, $S_{\text {max }}^{\prime}$ ) denote the maximum computation time associated with any processor (resp., source). By construction of the open-shop problem we have that:

$$
S_{\text {max }}^{\prime}=S_{\text {max }}
$$

where the quantity $S_{\max }$ for the original $S W A$ problem is defined in (3). Note, now, that an $\alpha$-approximation algorithm is used in Step $\mathbf{S} \mathbf{1}$ for the multiprocessor scheduling problem. This approximation algorithm respects constraint $\mathbf{C 1}$ only, therefore the optimal for the multiprocessor scheduling problem is at most equal to the optimal for the $S W A$ problem. Thus,

$$
D_{\max }^{\prime}=\alpha C_{\max }^{o p t}
$$

where $C_{m a x}^{o p t}$ refers to the $S W A$ problem. Since the Gonzalez and Sahni algorithm for the preemptive openshop scheduling problem is optimal [9], the schedule produced by the decomposition algorithm has length

$$
C_{m a x}^{D A}=\max \left\{D_{\max }^{\prime}, S_{\text {max }}^{\prime}\right\} \leq \alpha C_{\text {max }}^{\text {opt }}
$$

where the last inequality follows from (6). 
The above lemma implies that, if we use LPT (respectively, MULTI-FIT) in Step S1, then the decomposition algorithm is a 4/3-approximation (resp., 1.2-approximation) algorithm. On the other hand, the decomposition algorithm becomes a PTAS if the PTAS developed by Hochbaum and Schmoys for the multiprocessor scheduling problem is used in Step S1 to obtain the assignment of jobs to processors.

\section{Non-Preemptive Scheduling}

Let us now consider the non-preemptive $S W A$ problem, whereby, once an operation $O_{s d}$ has started processing on a certain processor $x$, it must be completed before the processor can start executing another operation. The following lemma proves that the non-preemptive version of the problem is also $\mathcal{N} \mathcal{P}$-complete.

Lemma 3 The non-preemptive SWA problem is NP-complete.

Proof. Consider an instance of the non-preemptive $S W A$ problem with $m$ processors and $n$ jobs. Each job $J_{d}, d=1, \cdots, n$, of the instance consists of a single operation $O_{d d}$ with non-zero processing time $p_{d d}$ (i.e., all other operations $O_{s d}, s \neq d, s=1, \cdots, n$, of job $J_{d}$ are such that $p_{s d}=0$ ). This instance consists of $n$ independent tasks (the $n$ operations $O_{d d}$ ) and $m<n$ processors. Thus, the well-known $\mathcal{N} \mathcal{P}$-complete multiprocessor scheduling problem $[6,11]$ is a special case of the non-preemptive $S W A$ problem.

\subsection{Algorithm Based on Problem Decomposition}

An algorithm based on a problem decomposition, similar to the one developed for the preemptive case in Section 3, can also be applied to this problem. However, non-preemptive open-shop scheduling is an $\mathcal{N} \mathcal{P}$ complete problem [9], but it is known that any list scheduling algorithm is a 2-approximation algorithm for this problem [13]. Therefore, instead of the Gonzalez and Sahni optimal algorithm for preemptive openshop scheduling, a 2-approximation list scheduling algorithm for the non-preemptive open-shop scheduling problem is applied in Step S2 of the decomposition algorithm DA in Section 3. We now have the following lemma.

Lemma 4 If an $\alpha$-approximation algorithm is applied to the corresponding multiprocessor scheduling problem in Step $\mathbf{S} 1$ of the decomposition, then the decomposition algorithm $\mathbf{D A}$ is a $2 \alpha$-approximation algorithm for the non-preemptive SWA problem.

Proof. Correctness. The proof of correctness is similar to the one given for the preemptive $S W A$ problem in Lemma 2.

Approximation Claim. If an $\alpha$-approximation algorithm is used in Step $\mathbf{S} \mathbf{1}$ for the multiprocessor scheduling problem, by construction of the open-shop problem we have that (refer also to the proof of Lemma 2):

$$
D_{\max }^{\prime}=\alpha C_{\max }^{o p t}
$$




$$
S_{\max }^{\prime}=S_{\max }
$$

where the quantity $S_{\max }$ for the original $S W A$ problem is defined in (3). Since list scheduling is a 2approximation algorithm for the non-preemptive open-shop scheduling problem [9], the schedule produced by the decomposition algorithm has length

$$
C_{\max }^{D A} \leq 2 \max \left\{D_{\max }^{\prime}, S_{\max }^{\prime}\right\} \leq 2 \alpha C_{\max }^{\text {opt }}
$$

where the last inequality follows from (5).

We now note that, while the decomposition algorithm DA will correctly schedule the operations of the non-preemptive $S W A$ problem (i.e., the resulting schedule will satisfy constraints $\mathbf{C 1}, \mathbf{C 2}$, and C3), it may actually do more than is needed to satisfy the non-preemption requirement. Consider jobs $J_{d}$ and $J_{d^{\prime}}, d \neq d^{\prime}$, if they exist, that have been assigned to the same processor $x$. In Step S2 of the algorithm, a new operation $O_{s x}, s=1, \cdots, n$, is created for the new open-shop problem, with a processing time equal to the sum of the processing times of operations $O_{s d}$ and $O_{s d^{\prime}}$ (and, possibly, the operations of other jobs assigned to the same processor) of the original $S W A$ problem. The list scheduling algorithm in Step S2 applied to the open-shop scheduling problem will ensure that the operation $O_{s x}$ will not be preempted, while the requirement for the original $S W A$ problem is simply that operations $O_{s d}$ and $O_{s d^{\prime}}$ not be preempted.

\subsection{Algorithms Based on List Scheduling}

We now present list scheduling algorithms for the non-preemptive $S W A$ problem. We first show that a naive implementation of list scheduling can produce schedules that are a factor of $m$ from the optimal schedule. We then describe a more refined version of list schedule that yields a 2-approximation algorithm for the non-preemptive $S W A$ problem.

We will need the following definitions in our discussion. A job $J_{d}$ is said to be assigned to processor $x$ at time $t$ if:

1. no operation $O_{s d}, s=1, \cdots, n$, has been executed (fully or partially) on any processor before time $t$, and

2. processor $x$ is idle at time $t$ and starts processing some operation $O_{s d}, s=1, \cdots, n$, at that time.

Because of the problem constraints, once a job $J_{d}$ is assigned to processor $x$, all operations $O_{s d}, s=1, \cdots, n$ must be executed on $x$. An operation $O_{s d}$ is called schedulable on processor $x$ at time $t$ if:

1. job $J_{d}$ has not been assigned to a different processor $y$ at some time $t^{\prime}<t$, and

2. an incompatible operation $O_{s^{\prime}} d^{\prime}$ (i.e., such that $s=s^{\prime}$ or $d=d^{\prime}$ ) is not being executed by another processor $y$ at time $t$.

When a processor $x$ becomes idle at some time $t$, it can only start processing an operation that is schedulable at time $t$. 


\subsubsection{List Scheduling, version 1}

Algorithm LS1 (List scheduling, version 1)

All the tasks $O_{s d}, s, d=1, \cdots, n$, are initially arranged in an arbitrary list $L$. Consider a processor $x$ which becomes idle at time $t$. If list $L$ is empty, the algorithm terminates. Otherwise, processor $x$ starts processing the first schedulable operation $O_{s d}$ in $L$, and the operation is removed from the list. If no schedulable operation is found in $L$, processor $x$ remains idle until time $t^{\prime}>t$ at which another processor $y$ that was busy at time $t$ completes its operation. At time $t^{\prime}$, processors $x$ and $y$ each scan list $L$ to select a new schedulable operation to process. (Ties are broken arbitrarily).

Lemma 5 Algorithm LS1 is an m-approximation algorithm for the non-preemptive SWA problem.

Proof. Correctness. By construction, the algorithm ensures that a processor may execute at most one operation at any time instant, thus satisfying constraint C3. The definition of a "schedulable" operation above, and the requirement that a processor, upon becoming idle, selects a schedulable operation for execution, also ensure that the algorithm will never violate constraints $\mathbf{C 1}$ or $\mathbf{C 2}$.

Approximation Claim. Let $C_{\max }^{L S 1}$ denote the makespan of a schedule produced by algorithm LS1, and let $T_{i d l e}$ denote the total idle time (over all $m$ processors) in this schedule. We first observe that at no point in time can all $m$ processors be idle in a schedule produced by using algorithm $\mathbf{L S 1}$, thus, $T_{\text {idle }} \leq(m-1) C_{\max }^{L S 1}$. Because of (4), we obtain:

$$
\begin{aligned}
C_{\max }^{L S 1} & \leq \frac{T_{i d l e}+T_{s e q}}{m} \leq \frac{m-1}{m} C_{\max }^{L S 1}+C_{\max }^{o p t} \\
& \Rightarrow C_{\max }^{L S 1} \leq m C_{\max }^{o p t} .
\end{aligned}
$$

The following problem instance shows that, asymptotically, the bound of the lemma is a tight one. The instance consists of a number $m$ of processors and a number $2 m$ of jobs whose operations are shown in Figure 1. The first $m$ jobs consist of two operations, one long operation of length $M-\frac{m+1}{m}$ and one short operation of length $\frac{1}{m}$. The last $m$ jobs consist of exactly $m$ short operations each, of length $\frac{1}{m}$. Let $M=m(m-1)$. The optimal schedule, shown in Figure 2(a), is such that processor $x, x=1, \cdots, m$, executes exactly two jobs, say jobs $x$ and $x+m$. This schedule has length $C_{\max }^{\text {opt }}=M=m(m-1)$. On the other hand, it is easy to see that under algorithm LS1, it is possible for one processor, say processor 1, to be assigned jobs 1 through $m+1$, while processor $x, x=2, \cdots, m$, is assigned only one of the last $m-1$ jobs. Such a schedule is illustrated in Figure 2(b), and has length

$$
C_{\max }^{L S 1}=2+m\left(M-\frac{m+1}{m}\right)=m M-(m-1)=m^{2}(m-1)-(m-1)
$$

Then,

$$
\frac{C_{\max }^{L S 1}}{C_{m a x}^{o p t}}=\frac{m^{2}(m-1)-(m-1)}{m(m-1)}=m-\frac{1}{m} .
$$




\begin{tabular}{|c|c|c|c|c|c|c|c|c|c|}
\hline & $J_{1}$ & $J_{2}$ & $J_{3}$ & $\cdots$ & $J_{m}$ & $J_{m+1}$ & $J_{m+2}$ & $\cdots$ & $J_{2 m}$ \\
\hline 1 & {$\left[M-\frac{m+1}{m}\right.$} & 0 & 0 & $\ldots$ & 0 & 0 & 0 & $\cdots$ & 0 \\
\hline 2 & $\frac{1}{m}$ & $M-\frac{m+1}{m}$ & 0 & $\ldots$ & 0 & 0 & 0 & $\ldots$ & 0 \\
\hline 3 & 0 & $\frac{1}{m}$ & $M-\frac{m+1}{m}$ & $\ldots$ & 0 & 0 & 0 & $\cdots$ & 0 \\
\hline$\vdots$ & $\vdots$ & $\vdots$ & $\vdots$ & $\vdots$ & $\vdots$ & $\vdots$ & $\vdots$ & $\vdots$ & $\vdots$ \\
\hline$m$ & 0 & 0 & 0 & $\cdots$ & $M-\frac{m+1}{m}$ & 0 & 0 & $\cdots$ & 0 \\
\hline$m+1$ & 0 & 0 & 0 & $\ldots$ & $\frac{1}{m}$ & $\frac{1}{m}$ & $\frac{1}{m}$ & $\ldots$ & $\frac{1}{m}$ \\
\hline$m+2$ & 0 & 0 & 0 & $\cdots$ & 0 & $\frac{1}{m}$ & $\frac{1}{m}$ & $\cdots$ & $\frac{1}{m}$ \\
\hline$\vdots$ & $\vdots$ & $\vdots$ & $\vdots$ & $\vdots$ & $\vdots$ & $\vdots$ & $\vdots$ & $\vdots$ & $\vdots$ \\
\hline $2 m$ & 0 & 0 & 0 & $\cdots$ & 0 & $\frac{1}{m}$ & $\frac{1}{m}$ & $\cdots$ & $\frac{1}{m}$ \\
\hline
\end{tabular}

Figure 1: Problem instance that asymptotically achieves the upper bound of Lemma 5

\subsubsection{List Scheduling, version 2}

We now present a different version of list scheduling which yields a 2-approximation algorithm for the nonpreemptive problem.

Algorithm LS2 (List scheduling, version 2)

The $n$ jobs $J_{d}, d=1, \cdots, n$, are initially arranged in a list $L$. The operations of each job $J_{d}$ are also arranged in a list $L_{d}$. A list $l_{x}$ is also associated with each processor $x$, and it is initialized to the empty list. Consider a processor $x$ that becomes idle at time $t$, and let $O_{s d}$ be the operation that was just completed by the processor. Processor $x$ selects an operation to process next by taking the following three steps in the order presented:

1. if there exists an operation $O_{s d^{\prime}}, d^{\prime} \neq d$, in the processor's list $l_{x}$, it is removed from the list and $x$ starts processing this operation at time $t$ (note that operation $O_{s d^{\prime}}$ is schedulable on $x$ at time $t$, since scheduling it does not violate constraint C2).

2. if no operation $O_{s d^{\prime}}$ is found in Step 1, $x$ starts processing any other schedulable operation in its list $l_{x}$, and the operation is removed from the list.

3. If no operation is found in the first two steps, list $L$ is scanned. If $L$ is empty, the algorithm terminates. Otherwise, let $J_{d^{\prime}}$ be the first job in $L$ with an operation that is schedulable on processor $x$ at time $t$, if one exists. Job $J_{d^{\prime}}$ is removed from $L$, and its list of operations $L_{d^{\prime}}$ is appended to processor $x$ 's list $l_{x}$. Processor $x$ starts processing the first schedulable operation in its new list $l_{x}$, and the operation is removed from $l_{x}$.

If no schedulable operation is found at the end of the third step, processor $x$ remains idle until time $t^{\prime}>t$ at which another processor $y$ that was busy at time $t$ completes its operation. At time $t^{\prime}$, processors $x$ 


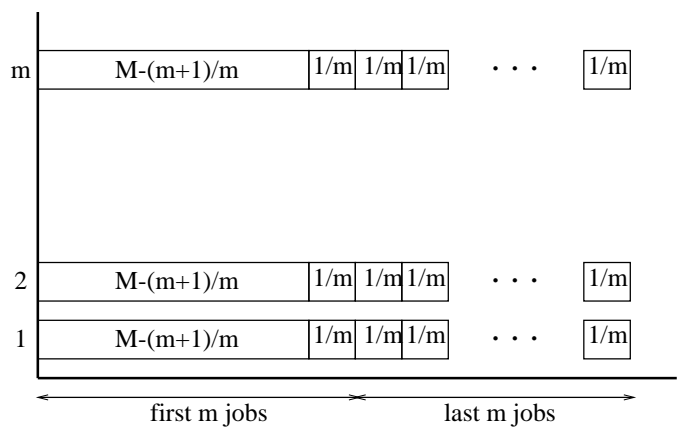

(a) Optimal schedule

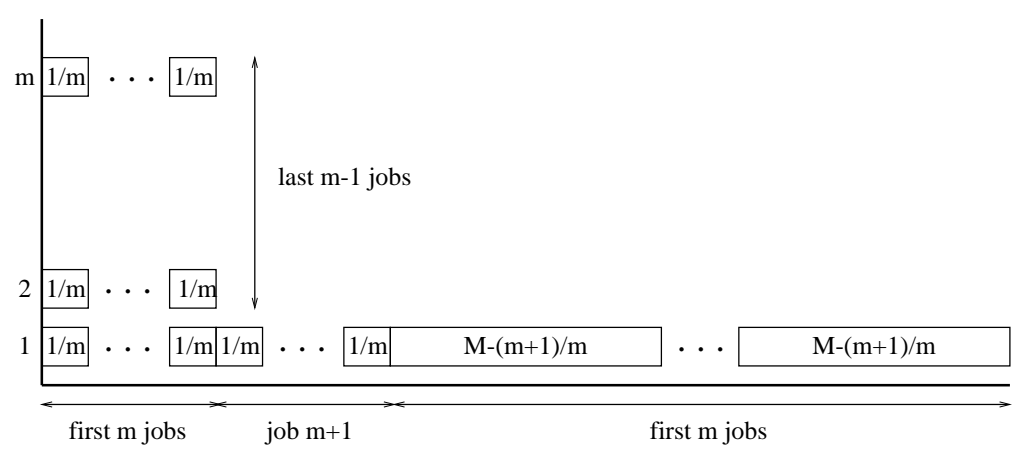

(b) Schedule under algorithm LS1

Figure 2: (a)Optimal schedule and (b) worst case schedule produced by algorithm LS1 for the instance shown in Figure 1

and $y$ each repeat the above procedure to select a new schedulable operation to process. (Ties are broken arbitrarily.)

Lemma 6 Algorithm LS2 is a 2-approximation algorithm for instances of the non-preemptive SWA problem for which no operation has zero processing time (i.e., $p_{s d}>0 \forall s, d$ ).

Proof. Correctness. By construction, the algorithm satisfies constraint C3. The fact that when the first operation of a job $J_{d}$ is schedulable on a processor $x$, the job is removed from list $L$ and is appended to processor $x$ 's list, ensures that constraint $\mathbf{C} 1$ is not violated. Finally, the requirement that a processor, upon becoming idle, selects a schedulable operation for execution, guarantees that the resulting schedule will satisfy constraint $\mathbf{C 2}$.

Approximation Claim. Consider a schedule $\mathcal{S}$ built using algorithm LS2, and let $J_{d}$ be the job that is the last to be assigned to a processor (i.e., all other jobs have been assigned to processors before $J_{d}$ ). Let $t$ be the time when job $J_{d}$ is assigned to a processor. We claim that no processor is idle before time $t$. To see that the claim is true, assume that a processor $y$ became idle at time $t^{\prime}<t$. Note now that at most $m-1$ different operations were being processed at time $t^{\prime}$, one at each of the other processors. By assumption, $J_{d}$ 
includes $n>m-1$ non-zero operations, and $n-m+1$ of these operations are schedulable on $y$ at time $t^{\prime}$. Thus, under algorithm LS2, job $J_{d}$ should have been assigned to $y$ at that point, contradiction.

At time $t$, all jobs have been assigned to processors. Let $\mathcal{P}=\left\{\mathcal{J}_{1}, \cdots, \mathcal{J}_{m}\right\}$ be the partition of the job set $\mathcal{J}$ such that $\mathcal{J}_{x}$ is the subset of jobs that have been assigned by the algorithm to processor $x, x=1, \cdots, m$. Let $p_{s d}(t)$ be the amount of processing that operation $O_{s d}$ has received up to time $t$. Consider a new scheduling problem with $m$ processors and $n$ jobs $J_{s}^{\prime}$, where each job is a set of operations, $J_{s}^{\prime}=\left\{O_{s 1}^{\prime}, \cdots, O_{s m}^{\prime}\right\}$, and the processing time of operation $O_{s x}^{\prime}$ is given by:

$$
p_{s x}^{\prime}=\sum_{d \in \mathcal{J}_{x}}\left(p_{s d}-p_{s d}(t)\right)
$$

This new problem is an instance of open-shop scheduling [9] with $m$ processors and $n$ jobs $J_{s}^{\prime}$, where each job has a single operation $O_{s x}^{\prime}$ to be executed on each processor $x$. We now observe that algorithm LS2 is also a list scheduling algorithm for this open-shop problem. To see this, consider a processor $x$ that becomes idle at time $t^{\prime}>t$. Since the last job was assigned to some processor at time $t$, no new jobs will be assigned to $x$ at or after time $t^{\prime}$. Let $O_{s d}$ be the operation that was just completed by $x$ at time $t^{\prime}$. Because of the first step in selecting a new job under algorithm LS2, if there is another job $O_{s d^{\prime}}$ in list $l_{x}, x$ will start processing $O_{s d^{\prime}}$ at time $t^{\prime}$. When $x$ becomes idle again, the same selection process will be repeated until all operations $O_{s d^{\prime}}$ with the same source $s$ in list $l_{x}$ are completed (note that each of these operations are schedulable on $x$ at the instant the previous one is completed). Thus, these operations $O_{s d^{\prime}}$ will be executed back-to-back, in some order, without interruption, and no operations with the same source will be added to list $l_{x}$ after time $t^{\prime}$. But all these operations $O_{s d^{\prime}}$ are part of the operation $O_{s x}^{\prime}$ of job $J_{s}^{\prime}$ in the open shop problem. Consequently, operation $O_{s x}^{\prime}$ will be executed without preemption on processor $x$ by algorithm LS2. Therefore, algorithm LS2 is a list scheduling algorithm for the open-shop problem.

Let $D_{\max }^{\prime}$ (respectively, $S_{\max }^{\prime}$ ) denote the maximum computation time associated with any processor (resp., source) in the open-shop problem. Since there is no idle time in the schedule constructed by algorithm LS2 until time $t$, we have that (note that we give the problem to which the various parameters are related in parentheses, $O S$ for open-shop, and $S W A$ for the original $S W A$ problem):

$$
C_{\max }^{\text {opt }}(S W A) \geq t+\max \left\{D_{\max }^{\prime}, S_{\max }^{\prime}\right\}
$$

Since LS2 is a list scheduling algorithm for the open-shop problem, we also have that:

$$
C_{\text {max }}^{L S 2}(O S) \leq 2 \max \left\{D_{\text {max }}^{\prime}, S_{\text {max }}^{\prime}\right\} \leq 2 C_{\max }^{\text {opt }}(O S)
$$

Finally, we get the desired result as follows:

$$
\begin{aligned}
C_{\text {max }}^{L S 2}(S W A) & =t+C_{\text {max }}^{L S 2}(O S) \leq t+2 \max \left\{D_{\text {max }}^{\prime}, S_{\text {max }}^{\prime}\right\} \\
& \leq t+C_{\text {max }}^{\text {opt }}(S W A)+\max \left\{D_{\text {max }}^{\prime}, S_{\text {max }}^{\prime}\right\} \leq 2 C_{\text {max }}^{\text {opt }}(S W A)
\end{aligned}
$$


Now assume that some of the operations in the original non-preemptive $S W A$ problem are zero. Without loss of generality, assume that each job $J_{d}$ consists of at least one operation of non-zero length (otherwise, we can remove this job reducing the problem into an equivalent one with $m$ processors and $n-1$ jobs). We can obtain a schedule that is at most twice the optimum one for this problem by taking the following three steps. First, replace all zero-length operations with ones of length equal to $\epsilon>0$. Then, run algorithm LS2 on this new instance of the $S W A$ problem for which no operation has zero processing time. Finally, remove from the schedule obtained by algorithm LS2 all operations that are of zero processing time in the original problem.

To see that this schedule is at most twice the optimum for the original $S W A$ problem (the one with some operation of zero length), we observe that at most $n(n-1)$ operations are added to the problem instance in the first step. Now note that a schedule for the new problem can be obtained by sequentially processing the $n(n-1)$ operations on one processor after the end of the optimal schedule for the original problem, thus:

$$
C_{m a x}^{o p t}(\text { new }) \leq C_{m a x}^{o p t}(\text { original })+n(n-1) \epsilon
$$

The optimum schedule for the new problem is at most equal to this schedule. Since at the third step of the algorithm we remove some operations from the schedule produced by LS2, we have that:

$$
\begin{aligned}
C_{\max }^{L S 2}(\text { original }) & \leq C_{\max }^{L S S 2}(\text { new }) \leq 2 C_{\max }^{\text {opt }}(\text { new }) \\
& \leq 2 C_{\max }^{\text {opt }}(\text { original })+2 n(n-1) \epsilon
\end{aligned}
$$

By selecting an appropriate value for $\epsilon$, we see that the schedule obtained in this way for the original problem is at most twice the optimal schedule plus a constant.

Finally, we note that algorithm LS2 is valid in an on-line context, where the jobs are not known in advance but appear one after the other.

\section{Concluding Remarks}

We considered the Scheduling and Wavelength Assignment (SWA) problem which has applications in packetswitched optical WDM networks. We proved that the SWA problem is $\mathcal{N} \mathcal{P}$-hard for both the preemptive and the non-preemptive cases. Furthermore, we propose two efficient approximation algorithms. For the preemptive case, we described an efficient approximation algorithm based on a natural decomposition of the problem. For the non-preemptive case, we presented two list scheduling algorithms, the second of which is a 2-approximation algorithm for both the off-line and the on-line contexts. 


\section{References}

[1] I. Baldine and G. N. Rouskas. Dynamic load balancing in broadcast WDM networks with tuning latencies. In Proceedings of INFOCOM '98, pages 78-85. IEEE, March 1998.

[2] I. Baldine and G. N. Rouskas. Dynamic reconfiguration policies for WDM networks. In Proceedings of INFOCOM '99, pages 313-320. IEEE, March 1999.

[3] G-S. Kuo (Ed.). Special issue on multiprotocol label switching. IEEE Communications Magazine, 37(12), December 1999.

[4] D. K. Friesen. Tighter bounds for the multifit processor scheduling algorithm. SIAM Journal on Computation, 13(1):170-181, 1984.

[5] M. Fujiwara, M. Goodman, M. O'Mahony, O. Tonguz, and A. Willner (Eds.). Special issue on multiple wavelength technologies and networks. Journal of Lightwave Technology, 14(6), June 1996.

[6] M. R. Garey and D. S. Johnson. Computers and Intractability. W. H. Freeman and Co., New York, 1979.

[7] O. Gerstel, B. Li, A. McGuire, G. N. Rouskas, K. Sivalingam, and Z. Zhang (Eds.). Special issue on protocols and architectures for next generation optical WDM networks. IEEE Journal Selected Areas in Communications, 18(10), October 2000. (To appear).

[8] T. Gonzalez. A note on open-shop preepmptive schedules. IEEE Transactions on Computers, C-28:782$786,1979$.

[9] T. Gonzalez and S. Sahni. Open shop scheduling to minimize finish time. Journal of the Association for Computing Machinery, 23(4):665-679, Oct 1976.

[10] R. L. Graham. Bounds on multiprocessing timing anomalies. SIAM Journal of Applied Mathematics, 17(2):416-429, Mar 1969.

[11] R. L. Graham, E. L. Lawler, J. K. Lenstra, and A. H. G. Rinnoy Kan. Optimization and approximation in deterministic sequencing and scheduling: A survey. Annals of Discrete Mathematics, 5:287-326, Jan 1979.

[12] D. S. Hochbaum and D. B. Schmoys. Using dual approximation algorithms for scheduling problems. Journal of ACM, 34(1):144-162, Jan 1987.

[13] T. Fiala I. Bárány. Tobbgepes, utemezesi problemak kozel optimalis megoldasa. Szigma-Mat.Kozgazdasagi Folyoirat, 15:177-191, 1982.

[14] E. L. Lawler and J. Labetoulle. On preemptive scheduling of unrelated parallel processors by linear programming. Journal of ACM, 25:612-619, 1978. 
[15] E. Modiano. WDM-based packet networks networks. IEEE Communications Magazine, 37(3):130-135, March 1999.

[16] R. Ramaswami and K. N. Sivarajan. Optical Networks. Morgan Kaufmann Publishers, San Francisco, California, 1998.

[17] G. N. Rouskas and V. Sivaraman. Packet scheduling in broadcast WDM networks with arbitrary transceiver tuning latencies. IEEE/ACM Transactions on Networking, 5(3):359-370, June 1997.

[18] G. N. Rouskas and V. Sivaraman. A reservation protocol for broadcast WDM networks and stability analysis. Computer Networks, 32(2):211-227, February 2000.

[19] V. Sivaraman and G. N. Rouskas. HiPeR- $\ell$ : A High Performance Reservation protocol with $\ell$ look-ahead for broadcast WDM networks. In Proceedings of INFOCOM '97, pages 1272-1279. IEEE, April 1997. 